\title{
Análisis numérico de los perfiles de velocidad de un flujo de agua a través de una tubería con reducción gradual
}

\section{(Numerical analysis of the speed profiles of a water flow through a gradual reduction pipe)}

\author{
Luis Fernando Toapanta Ramos ${ }^{1}$, Gabriel Alejandro Bohórquez Peñafiel ${ }^{1}$, Luis Eduardo \\ Caiza Vivas ${ }^{1}$, William Quitiaquez Sarzosa ${ }^{1}$
}

\begin{abstract}
Resumen:
El objetivo de este trabajo de investigación es comprender el comportamiento del flujo del agua a través de la contracción gradual. La dinámica de fluidos computacional (CFD), es un enfoque útil para resolver las ecuaciones que describen el movimiento de los fluidos, mediante métodos numéricos y técnicas computacionales; esta área de la mecánica proporciona los fundamentos de la hidráulica de tuberías e hidráulica de canales. Se ha realizado un estudio del flujo a través de la tubería para calcular las pérdidas en los cambios de la geometría. Las pérdidas de energía debido al cambio transversal del área, es una parte importante para analizar el flujo por medio de las tuberías. Se determinó los perfiles de velocidad debido al cambio de sección en los sistemas de tuberías. Se emplearon cálculos mediante el uso del modelo K-épsilon del software. Esta simulación proporciona los valores de los contornos de presión, velocidad y turbulencia cinética en varias secciones de la tubería donde el agua es el fluido.
\end{abstract}

Palabras clave: CFD; ANSYS fluent; contracción gradual; presión; perfiles de velocidad; turbulencia cinética.

\begin{abstract}
:
The objective of this research work is to understand the flow behavior of water through gradual contraction. Computational fluid dynamics (CFD) is a useful approach to solve the equations that describe the movement of fluids, using numerical methods and computational techniques; this area of mechanics provides the fundamentals of pipe hydraulics and channel hydraulics. A study of the flow through the pipeline has been made to calculate the losses in the geometry changes. The energy losses due to the transverse change of the area, is an important part to analyze the flow through the pipes. The velocity profiles were determined due to the change of section in the piping systems. Calculations were used using the K-epsilon software model. This simulation provides the values of the contours of pressure, velocity and kinetic turbulence in several sections of the pipe where the water is the fluid.
\end{abstract}

Keywords: CFD; ANSYS fluent; gradual contraction; Pressure; speed profiles; kinetic turbulence.

\section{Introducción}

La importancia de estimar los perfiles de velocidad y las pérdidas de energía en dispositivos de reducción del diámetro o reducciones graduales y codos, está dada por su utilización en sistemas a presión o en régimen forzado, tales como: líneas de conducción en plantas industriales (Anaya-Durand, Cauich-Segovia, Funabazama-Bárcenas, \& GraciaMedrano-Bravo, 2014), redes internas de distribución en centrales geotérmicas ( $F, 2000)$,

\footnotetext{
1 Universidad Politécnica Salesiana, Grupo de Investigación en Energías Renovables e Implementación Mecánica de Pymes (GIERIMP), Quito, Ecuador ( \{ltoapanta, wquitiaquez\} @ups.edu.ec; \{gbohorquezp, lcaizav1\} @est.ups.edu.ec ).
} 
conductos de centrales hidroeléctricas (Elbatran, Yaakob, \& Ahmeda, 2015), redes de distribución de agua a poblaciones (Yildirim \& Shing, 2010), sistemas de riego (Sesma, Molina-Martínez, Cavas-Martínez, \& Fernández-Pacheco, 2015), entre otros.

Las pérdidas de energía en los dispositivos de reducción del diámetro o reducciones graduales ocurren cuando el fluido circula por un conducto de diámetro constante y su sección transversal decrece de forma repentina o gradual, en dicha condición se presenta una transición suave de un diámetro mayor hacia uno menor. El fluido es encausado suavemente hacia un área menor esto permite que la velocidad de este incremente a medida que decrece el área transversal (Franzini \& Finnemore, 1999), esta pérdida es la que se da en el sistema, al unir dos tuberías de distinto diámetro, generalmente estos cambios de diámetro es necesario hacer al realizar los cálculos para el diámetro económico, que en la mayoría de los casos se obliga a usar dos series comerciales distintas. Esta unión se puede realizar con dos accesorios distintos, una contracción brusca o una gradual. En el caso gradual, la pérdida se ve afectada por la brusquedad de la reducción del diámetro, que se determina por el ángulo de reducción, para que el valor de la pérdida no sea grande, el ángulo de reducción no debe exceder de un valor especificado 80 ( Garzón Cruz, 2014). El flujo queda completamente limitado por la superficie sólida, en tuberías y en ductos. Al considerar un flujo compresible e incompresible a través de un tubo de sección transversal circular, este es uniforme a la entrada del tubo y su velocidad es igual a cero (Unet, 2016). El perfil de velocidad uniforme es igual a la velocidad media del fluido, se deforma gradualmente hasta alcanzar un perfil característico de un flujo laminar permanente en un tubo recto circular ('flujo de Poiseuille') (Montilva, 2009). La constante investigación sobre las pérdidas de energía que ocurren en estos dispositivos valida su importancia (Binding, Phillips, \& Phillips, 2006).Se realizaron muchos estudios numéricos para estimar la pérdida de energía a través de contracciones, expansiones y combinaciones de estas, mediante un fluido de agua (Pedros, 2010) .

Este fenómeno fue estudiado teóricamente por (Schiller, 1992); al considerar al flujo compuesto de dos regiones: una capa límite cercana a la pared del tubo y un núcleo de fluido libre de vorticidad, en que las fuerzas viscosas son despreciables a comparación con las fuerzas inerciales, con la suposición de un perfil de velocidad parabólico en la capa límite, con el empleo de la ecuación de Bernoulli en el núcleo y el método de Pohlhausen, predijo el crecimiento de la velocidad axial como una función de la distancia radial y de la distancia axial del fluido con baja medida desde la entrada al tubo.

En una de las publicaciones de (Fester, Mbiya, \& Slatter, 2008) investigó con fluidos no newtonianos las pérdidas de energía en contracciones graduales, con el uso de relaciones de diámetros de 0,22, 0,50 y 0,85, y números de Reynolds de 0,01 a 100.000, bajo la finalidad de corroborar y ampliar los datos existentes, se presentan un conjunto de resultados experimentales y un modelo numérico validado. De igual forma, (Rend, Sparrow, Bettenhausen, \& Abraham, 2013) hicieron un estudio numérico para determinar la pérdida de energía que se genera en una ampliación gradual utilizada en un sistema de tuberías; se dedujo que la caída de presión incrementa monótonamente al aumentar el ángulo de dilatación y que, en general, la caída de presión para un ángulo de dilatación dado decrece con el aumento del número de Reynolds y tiende a estabilizarse conforme este aumenta (Bae \& Kim, 2014).

Los objetivos del trabajo son simular mediante CFD el perfil de velocidad en tuberías con reducción graduales, empleando agua como sustancia principal. En la actualidad, la alternativa que se está considerando con mayor frecuencia para el estudio del flujo es el empleo de los métodos numéricos en computadoras.

La metodología CFD (Computational Fluid Dynamics) (Villaroel Quinde, 2015) es un método numérico que consiste en dividir el sistema de interés en volúmenes pequeños en donde se resuelven, de forma aproximada, las ecuaciones generales que describen el comportamiento del fluido. Esto permite obtener resultados en cada punto del sistema y evaluar de forma más rápida cualquier cambio en las condiciones del mismo. 


\section{Metodología}

\subsection{Descripción de la sección de análisis}

En este trabajo se realiza con la ayuda de una simulación en CFD, como fluido del proceso se toma al agua, en fase liquida a $20^{\circ} \mathrm{C}$, en una tubería de acero comercial cédula 40 con una reducción gradual. El estudio crítico se va a realizar en una tubería horizontal, en la que se conocen las condiciones de diseño. La tubería está formada por dos secciones, en la sección mayor con un diámetro nominal de $50 \mathrm{~mm}$ "D", y una longitud "L/2", y la sección de diámetro menor, "d", es de $25 \mathrm{~mm}$ de diámetro nominal, con una

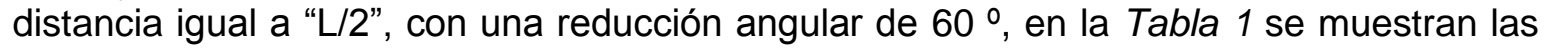
dimensiones. Adicional a esto se observa en la Figura 1 el esquema de la sección de análisis.

Tabla 1.Dimensiones de tubería de acero comercial cédula 40.

Fuente: (Mott, 2006)

\begin{tabular}{|l|l|l|l|l|l|}
\hline Sección & $\begin{array}{l}\text { Diámetro } \\
\text { nominal } \\
(\mathbf{m})\end{array}$ & $\begin{array}{l}\text { Diámetro } \\
\text { exterior } \\
(\mathbf{m})\end{array}$ & $\begin{array}{l}\text { Diámetro } \\
\text { interior } \\
(\mathbf{m})\end{array}$ & $\begin{array}{l}\text { Longitud } \\
\mathbf{L} / \mathbf{2} \\
(\mathbf{m})\end{array}$ & $\begin{array}{l}\text { Reducción } \\
\text { Gradual } \\
(\boldsymbol{\theta})\end{array}$ \\
\hline $\mathrm{D}$ & 0.05 & 0.0603 & 0.0525 & 0.5 & --- \\
\hline $\mathrm{D}$ & 0.025 & 0.0334 & 0.0266 & 0.5 & --- \\
\hline$\Theta$ & --- & --- & --- & --- & $60 \cong$ \\
\hline
\end{tabular}

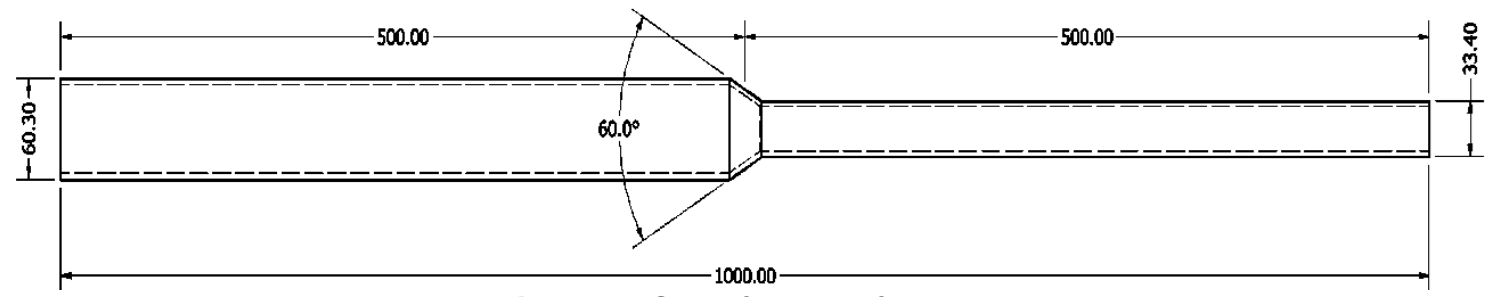

Figura 1. Sección de análisis.

\subsection{Descripción del flujo}

El problema trata de un flujo incompresible (líquido) y viscoso con densidad constante e isotermo, en este caso el agua. El tipo de flujo que se presenta es el movimiento de un fluido por el conducto; es muy importante en los problemas de dinámica de fluidos. Cuando se mueven por un canal cerrado de cualquier área de corte transversal, se puede presentar cualquiera de los dos tipos, dependiendo de las condiciones existentes.

Estos dos tipos de flujo pueden verse con frecuencia en un río o en cualquier corriente abierta. Cuando la velocidad es baja, su desplazamiento es uniforme y terso.

Sin embargo, cuando la velocidad es bastante alta, se observa una corriente inestable en la que se forman remolinos o pequeñas partículas de fluido que se mueven en todas las direcciones y con una gran diversidad de ángulos con respecto a la dirección normal del flujo. 


\subsection{Pérdidas de energía}

Las pérdidas de energía en tuberías estudiadas (Bariviera, Frizzone, \& Rettore, 2013) se clasifican como: de reducción brusca y de reducción gradual. Asimismo, los autores exponen que las pérdidas de energía locales se determinan mediante la Ecuación 1.

$$
h_{L}=K \frac{V^{2}}{2 g}
$$

Donde:

$h_{L}$ es la pérdida de carga $(\mathrm{m}) ; V$ es la velocidad media de circulación en el conducto de diámetro reducido $(\mathrm{m} / \mathrm{s}) ; \mathrm{g}$ es la aceleración de la gravedad $\left(\mathrm{m} / \mathrm{s}^{2}\right) ; K$ es un coeficiente de pérdidas del dispositivo (adimensional), que se obtiene mediante tablas o gráficos provenientes de resultados experimentales y que a menudo se conjugan con ecuaciones empíricas (Bae \& Kim, 2014).

En una reducción gradual del diámetro, de acuerdo con (Mataix, 2010), el coeficiente $K$ de este dispositivo (Ecuación 2) se determina con la utilización de un gráfico que esté en función del ángulo de reducción $(\theta)$ y de la relación del diámetro mayor con respecto al diámetro menor (D/d). (Fox, Pritchard, \& \& Mcdonald, 2006) exponen que el coeficiente de esta reducción se obtiene de valores tabulados, en función del ángulo de reducción $(\theta)$ y de la relación del área menor con respecto a la mayor $\left(A_{2} / A_{1}\right)$.

$$
K_{R G}=a+b(D / d)^{0.5}+c[\ln (D / d)] /(D / d)^{2}
$$

Donde:

$K_{R G}$, es el coeficiente de pérdidas de una reducción gradual del diámetro; $D / d$ es el valor de la relación del diámetro mayor con respecto al diámetro menor del dispositivo; $a, b, c$ son coeficientes que dependen del ángulo de la reducción $(\theta)$.

$$
\begin{aligned}
& a=-0.046121351-0.000029224 \theta^{2}+8.9116 \times 10^{-10} \theta^{4}-1.1811 \times 10^{-10} \theta^{6} \\
& b=0.046264338+0.000029251 \theta^{2}-9.2469 \times 10^{-10} \theta^{4}+1.2761 \times 10^{-10} \theta^{6} \\
& b=0.1503115+0.000064686 \theta^{2}-7.7480 \times 10^{-10} \theta^{4}-7.2780 \times 10^{-10} \theta^{6}
\end{aligned}
$$

\subsection{Contracción gradual}

La pérdida de energía en una contracción puede disminuir en forma sustancial si esta se hace más gradual. La Figura 2 muestra tal contracción formada por una sección cónica entre los dos diámetros, con bordes afilados en las uniones. El ángulo $\theta$ se denomina ángulo del cono.

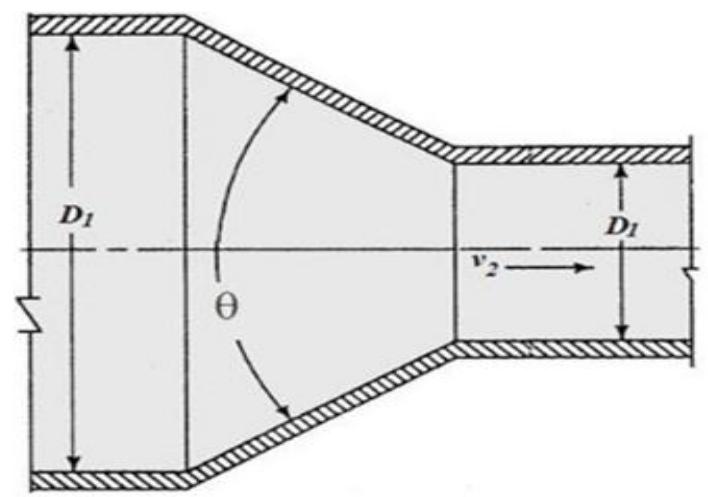

Figura 2. Diagrama de una contracción gradual. Fuente: (Mott, 2006). 
Conforme el ángulo del cono de la contracción disminuye a menos de $15^{\circ}$, el coeficiente de resistencia en realidad se incrementa. La razón es que los datos incluyen los efectos, tanto de la turbulencia local que ocasiona la separación del flujo como los de la fricción con el tubo. Para ángulos del cono más pequeños, la transición entre los dos diámetros es muy larga, lo cual aumenta las pérdidas por fricción

La razón es que los datos incluyen los efectos tanto de la turbulencia local ocasionada por la separación del flujo como de la fricción del conducto. Para los ángulos de cono menor, la transición entre los dos diámetros es muy larga, lo cual incrementa las pérdidas de fricción.

\subsection{Modelos de turbulencia}

Dadas las características del flujo, se hace necesario incluir un modelo de turbulencia. Los modelos de turbulencia más usados son los llamados "modelos de dos ecuaciones" $\kappa-\varepsilon, \kappa-\omega$. debido a que ofrecen una buena relación entre costo computacional y precisión en los resultados (ANSYS). Estos modelos resuelven las escalas de longitud y de velocidad mediante dos ecuaciones separadas, por eso su nombre.

Dentro de esta familia de modelos de turbulencia existen dos modelos principales: k$\varepsilon$ y k- $\omega$. Ambos usan la hipótesis de un gradiente de difusión para relacionar los esfuerzos de Reynolds con los gradientes medios de velocidad y la viscosidad turbulenta (Menter, 1994). La viscosidad turbulenta es modelada como el producto entre la velocidad turbulenta y la escala de longitud turbulenta.

El modelo de turbulencia $k-\varepsilon$ se ha vuelto útil en muchos cálculos prácticos de flujo de ingeniería. La precisión económica, robusta y razonable de este modelo de turbulencia explica su popularidad en simulaciones de flujo industrial y transferencia de calor. Es un modelo semiempírico y la derivación de las ecuaciones modelo se basa en consideraciones fenomenológicas y empíricas.

El modelo $\mathrm{k}-\varepsilon$ estándar es un modelo semiempírico basado en ecuaciones de transporte modelo para la energía cinética de turbulencia $(k)$ y su tasa de disipación $(\varepsilon)$. La ecuación de transporte modelo para $k$ se deriva de la ecuación exacta, mientras que la ecuación de transporte modelo para $\varepsilon$ se obtuvo con el razonamiento físico y tiene poca semejanza con su contraparte matemáticamente exacta. En la derivación del modelo k- $\varepsilon$, se consideró que el flujo es completamente turbulento, y los efectos de la viscosidad molecular son insignificantes (Bardina, Huang, \& Coakley, 1997). El modelo k - $\varepsilon$ estándar es por lo tanto válido solo para flujos turbulentos.

La energía cinética de la turbulencia $k$, y su velocidad de disipación $\varepsilon$, se obtienen a partir de las ecuaciones de transporte 3 y 4 :

$$
\begin{gathered}
\frac{\partial}{\partial}(\rho k)+\frac{\partial}{\partial x_{i}}\left(\rho k u_{i}\right)=\frac{\partial}{\partial x_{j}}\left[\left(\mu+\frac{u_{t}}{\sigma_{k}}\right) \frac{\partial k}{\partial x_{j}}\right]+G_{k}+G_{b}-\rho \varepsilon-Y_{M}+S_{k} \\
\frac{\partial}{\partial t}(\rho \varepsilon)+\frac{\partial}{\partial x_{i}}\left(\rho \varepsilon u_{i}\right)=\frac{\partial}{\partial x_{j}}\left[\left(\mu+\frac{u_{t}}{\sigma_{\varepsilon}}\right) \frac{\partial \varepsilon}{\partial x_{j}}\right]+C_{1 \varepsilon} \frac{\varepsilon}{k}\left(G_{k}+C_{3 \varepsilon} G_{b}\right)-\rho C_{2 \varepsilon} \frac{\varepsilon^{2}}{k}+S_{\varepsilon}
\end{gathered}
$$

En estas ecuaciones, $G_{k}$ representa la generación de energía cinética de turbulencia debido a los gradientes de velocidad promedio. $G_{b}$ es la generación de energía cinética de turbulencia debido a la flotabilidad. La cantidad $Y_{M}$ simboliza la contribución de la dilatación de la fluctuación a la turbulencia incompresible a la tasa de disipación global. $C_{1 \varepsilon}, C_{2 \varepsilon}$ y $C_{3 \varepsilon}$ son constantes. $\sigma_{k}$ y $\sigma_{\varepsilon}$ son el número de Prandtl turbulento para $k$ y $\varepsilon$, respectivamente. $S_{k}$ y $S_{\varepsilon}$ son términos fuente definidos por el usuario.

La viscosidad turbulenta, $u_{t}$, se calcula combinando $k$ y $\varepsilon$ según la Ecuación 5 :

$$
u_{t}=\rho C_{u} \frac{k^{2}}{\varepsilon}
$$


Donde:

$C_{u}$ es una constante, las constantes del modelo, $C_{1 \varepsilon}, C_{2 \varepsilon}, C_{u}, \sigma_{k}$ y $\sigma_{\varepsilon}$ tienen los siguientes valores predeterminados, tomados del software de simulación ANSYS V. 16.0.

$$
C_{1 \varepsilon}=1.44, C_{2 \varepsilon}=1.92, C_{u}=0.09, \sigma_{k}=1.0, \sigma_{\varepsilon}=1.3
$$

\begin{tabular}{|cl|}
\hline & Nomenclatura \\
$\rho$ & Densidad del fluido \\
$\tau$ & Tensor de esfuerzos de Reynolds \\
$k$ & Energía cinética de turbulencia \\
$\varepsilon$ & Tasa de disipación de turbulencia \\
$v_{T}$ & Viscosidad de arremolinamiento cinética \\
$S_{i j}$ & Tensor de velocidad de deformación \\
$G_{k}$ & Generación de energía cinética de turbulencia debido a la velocidad \\
$G_{b}$ & Generación de energía cinética de turbulencia debido a la flotabilidad \\
$\sigma_{k}$ & Número de Prandtl turbulento $\mathrm{k}$ \\
$\sigma_{\varepsilon}$ & Número de Prandtl turbulento $\varepsilon$ \\
$Y_{M}$ & Contribución de dilatación fluctuante \\
$u_{t}$ & Viscosidad turbulenta computada \\
$C_{10}, C_{2}, C_{1 \varepsilon}, C_{3 \varepsilon}$ & Constantes \\
\hline
\end{tabular}

\subsection{Mallado en flujo turbulento}

A través de la tubería se modela el flujo numéricamente mediante el software ANSYS fluent; en este trabajo se espera comprobar, el efecto de la turbulencia y perfiles de velocidad la tubería de acero comercial cédula 40, el modelo que se analizó es: turbulencia $\kappa-\varepsilon$ estándar. El fluido con el cual se trabajó fue agua líquida en toda la fase de la tubería. Como ya se mencionó, las simulaciones se realizaron con fluent, además, de elaborarlas en 2D. En la Figura 3, se aprecia el mallado de toda la línea de tubería, con un mallado fino que proporciona elementos cuadriláteros deseables.

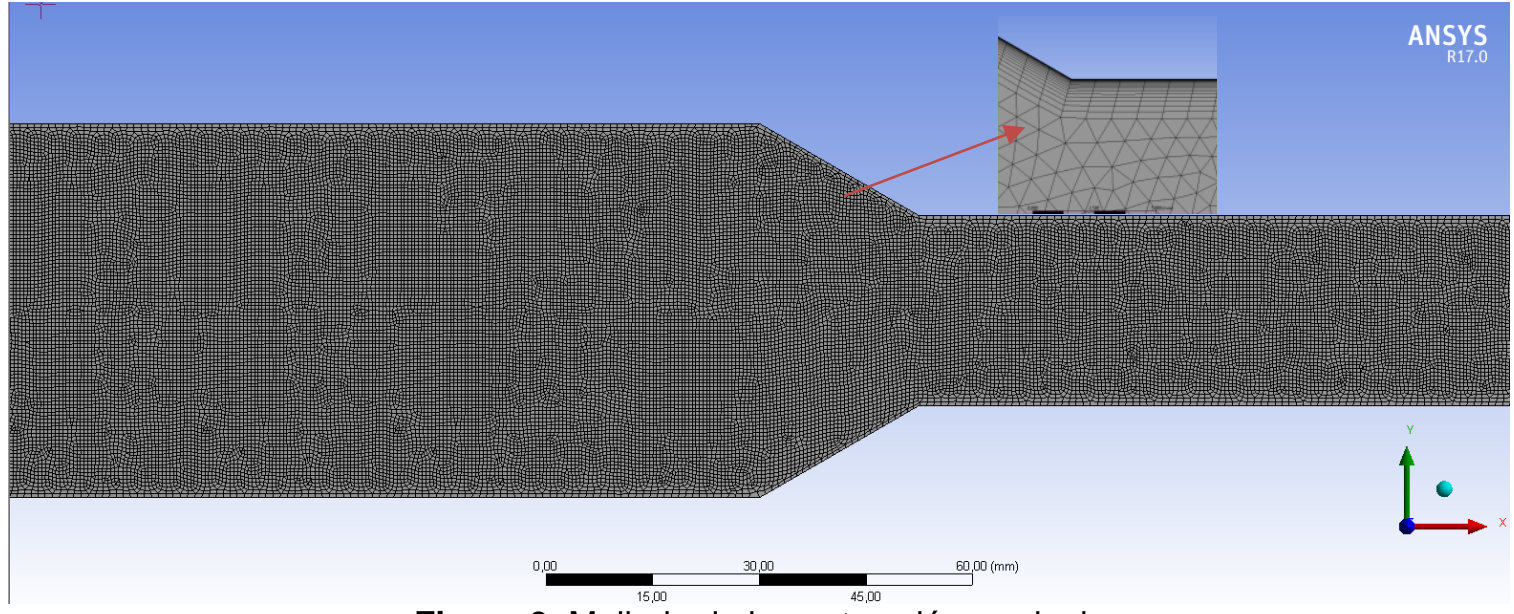

Figura 3. Mallado de la contracción gradual

En el estudio realizado se van a analizar diferentes tipos de variables, como la presión, la velocidad y la turbulencia cinética durante toda la línea de tubería, los lineamientos del mallado final se obtuvieron luego de varias simulaciones con distinto tipo de mallado, en la Figura 4 se observa la convergencia de mallado a utilizar en este proyecto; para ello se usó la herramienta Skewness, la cual acentúa la cantidad de elementos que forman el mallado; si la mayoría de estos se encuentran entre 0 - 0.25 el mallado está convergiendo, tal como se aprecia en la Figura 4. 


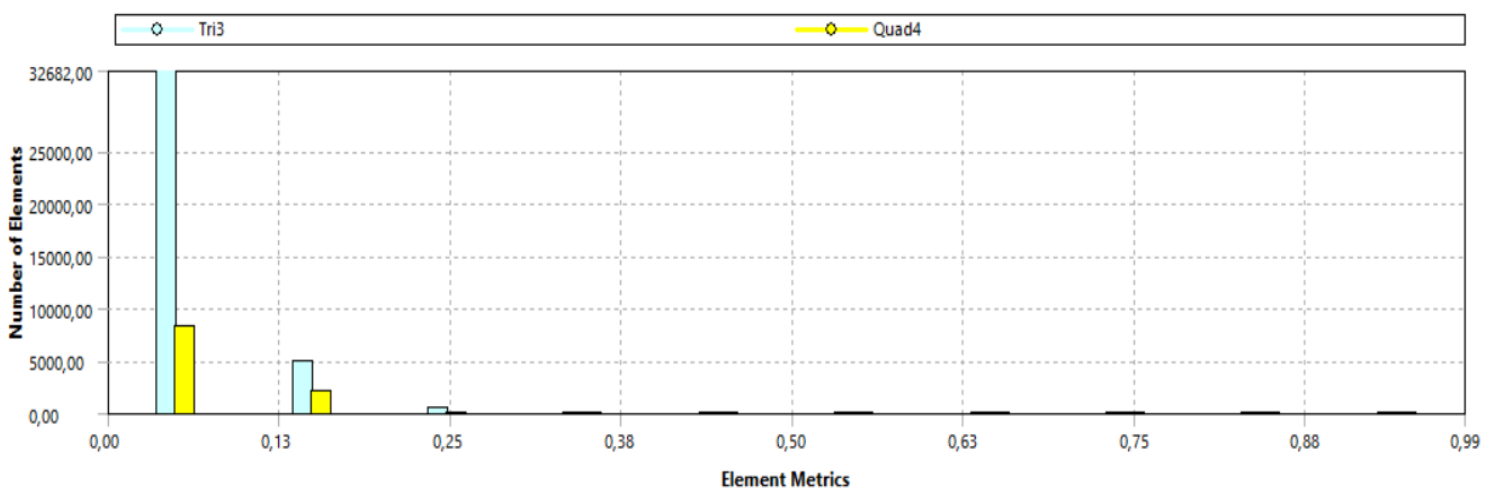

Figura 4. Convergencia del mallado

Entonces, un mallado ideal, es aquel que tiene un refinamiento infinito, por esta razón se utilizaron varias herramientas como: fase sizing, la cual determina el tamaño de la malla, inflation para refinar el mallado en las secciones importantes de análisis, conjuntamente con la calidad de la malla final mediante el método proximity and curvature.

Un aspecto importante en CFD es la independencia de malla, esta se fundamentó en las velocidades del fluido, inicial y final, en la Figura 5, se aprecian tres distintos perfiles de velocidad en función del tamaño de mallado; 0.5, 2.5, y $5 \mathrm{~mm}$ respectivamente, en donde el mallado fino otorga un perfil renovado de la velocidad del flujo.

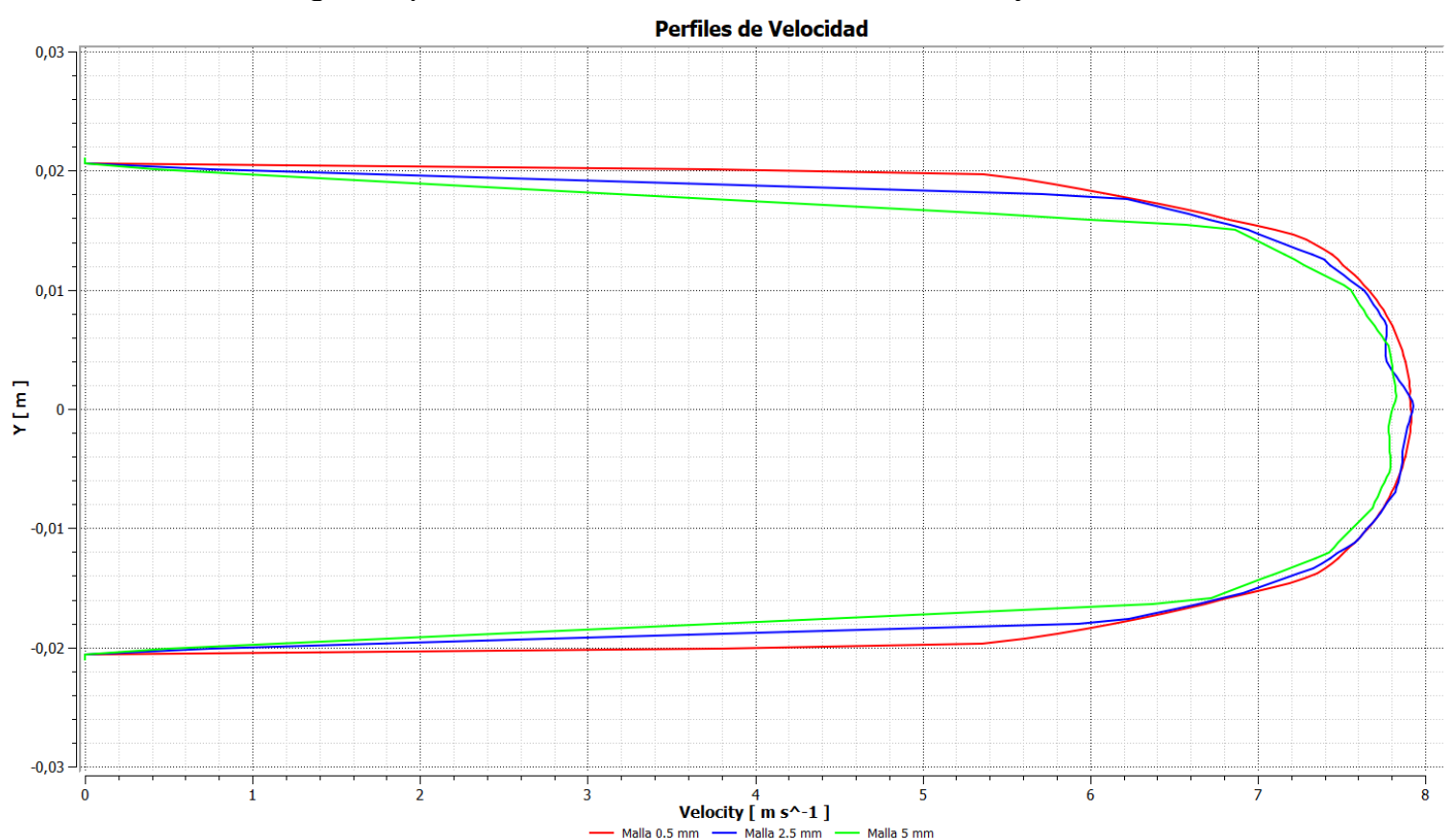

Figura 5. Independencia de malla, respecto a la velocidad

\section{Resultados y discusión}

\subsection{Simulación numérica del flujo}

Se inicia la simulación de un fluido turbulento (agua líquida) con el modelo de turbulencia $\kappa-\varepsilon$ estándar. La velocidad de entrada para toda la fase de la tubería es de $5,5 \mathrm{~m} / \mathrm{s}$. En la Figura 6 se observan los contornos de velocidad obtenidos a causa de la

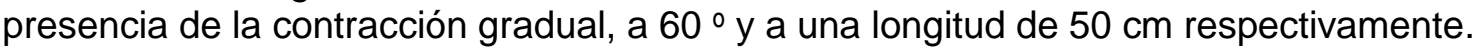




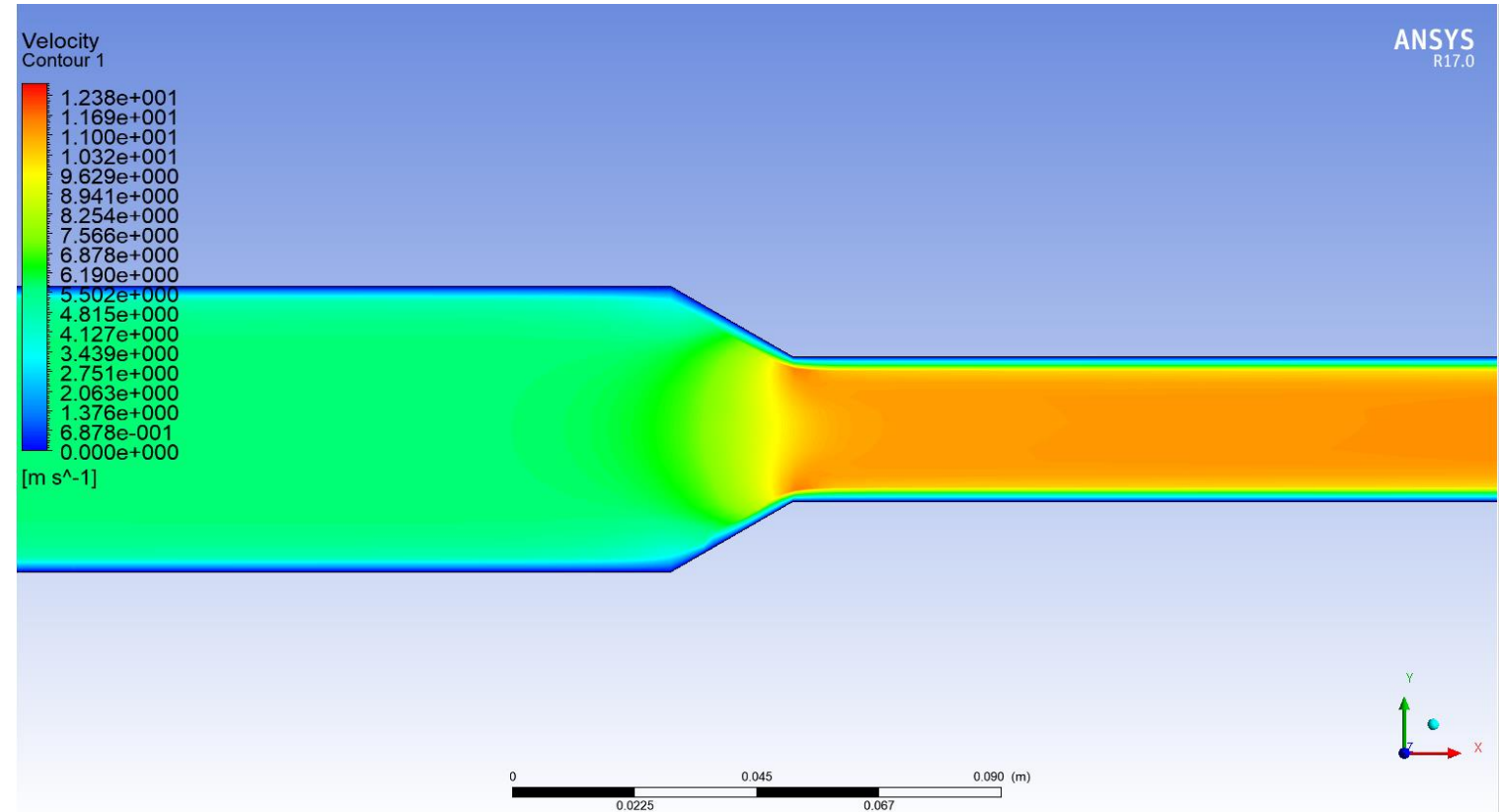

Figura 6. Velocidad, en la contracción gradual

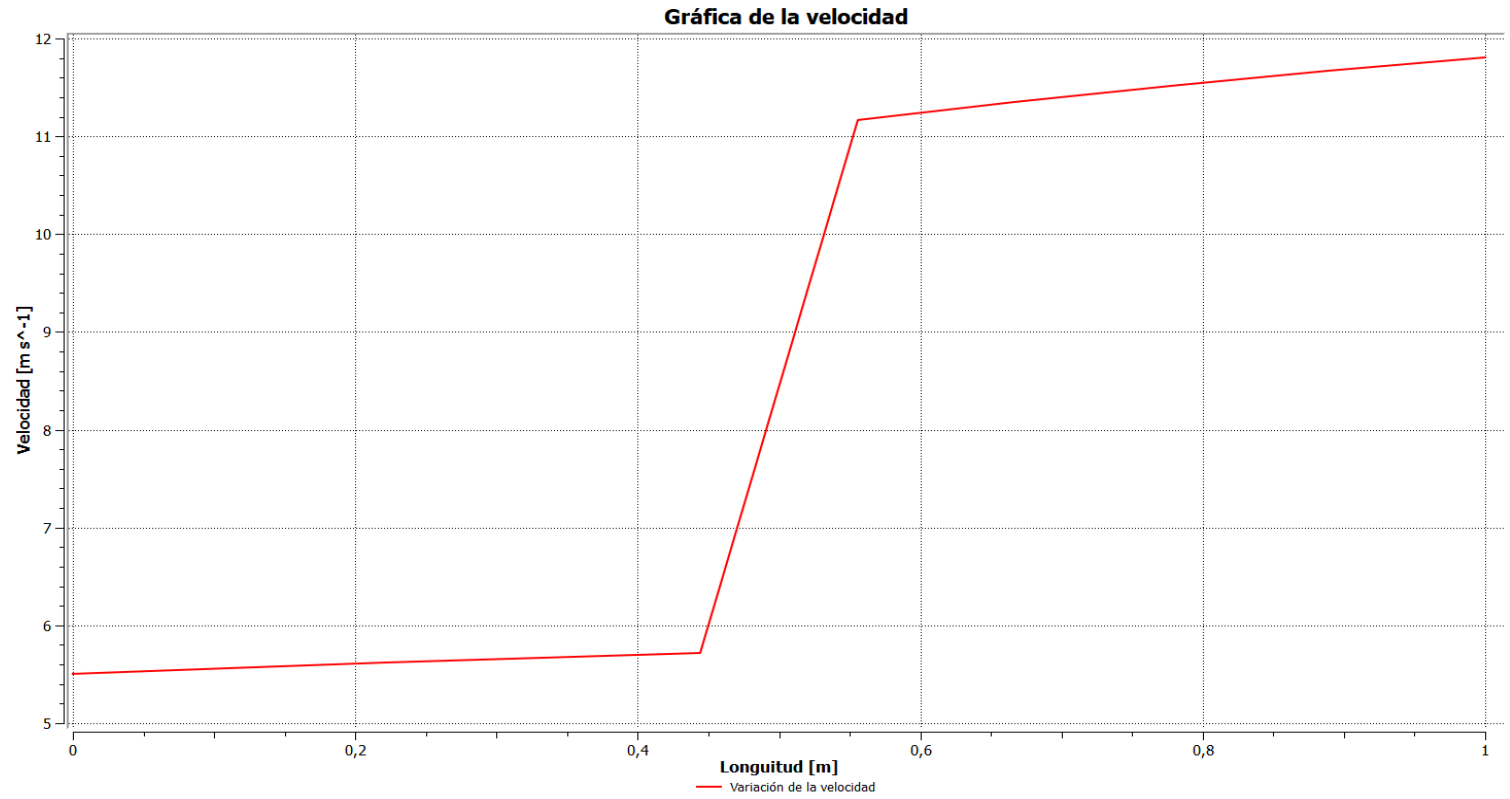

Figura 7. Variación de la velocidad

Se puede observar en la Figura 7 que la velocidad de entrada permanece casi constante antes y después de la contracción gradual, pero, al chocar el fluido con la misma, vemos un cambio drástico al reducir el área transversal de dicha tubería; en las partes superior e inferior de la figura, la velocidad va en aumento y toma un rango de 5.5 a 11.2 $\mathrm{m} / \mathrm{s}$.

Como complemento en la Figura 8 sus respectivos vectores de velocidad. Se visualiza que la velocidad aumenta si se toman valores de entre 9 y $12 \mathrm{~m} / \mathrm{s}$ en el sector de la contracción, provocando incremento del $140 \%$ en relación con la velocidad inicial.

Para el análisis de la turbulencia se toman las mismas consideraciones que se aplicaron anteriormente, tanto en velocidad como en el modelo de turbulencia, los resultados se aprecian en la Figura 9 y Figura 10. Los lugares donde existe mayor turbulencia son en la parte superior e inferior de la contracción gradual, con valores mínimos y máximos de 0.006 y $1.1 \mathrm{~m}^{2} / \mathrm{s}^{2}$ respectivamente, además, los efectos de 
turbulencia, a la salida de la contracción, podrían provocar efectos adversos en la pared de la tubería como, desgaste y desprendimiento de porciones de material.

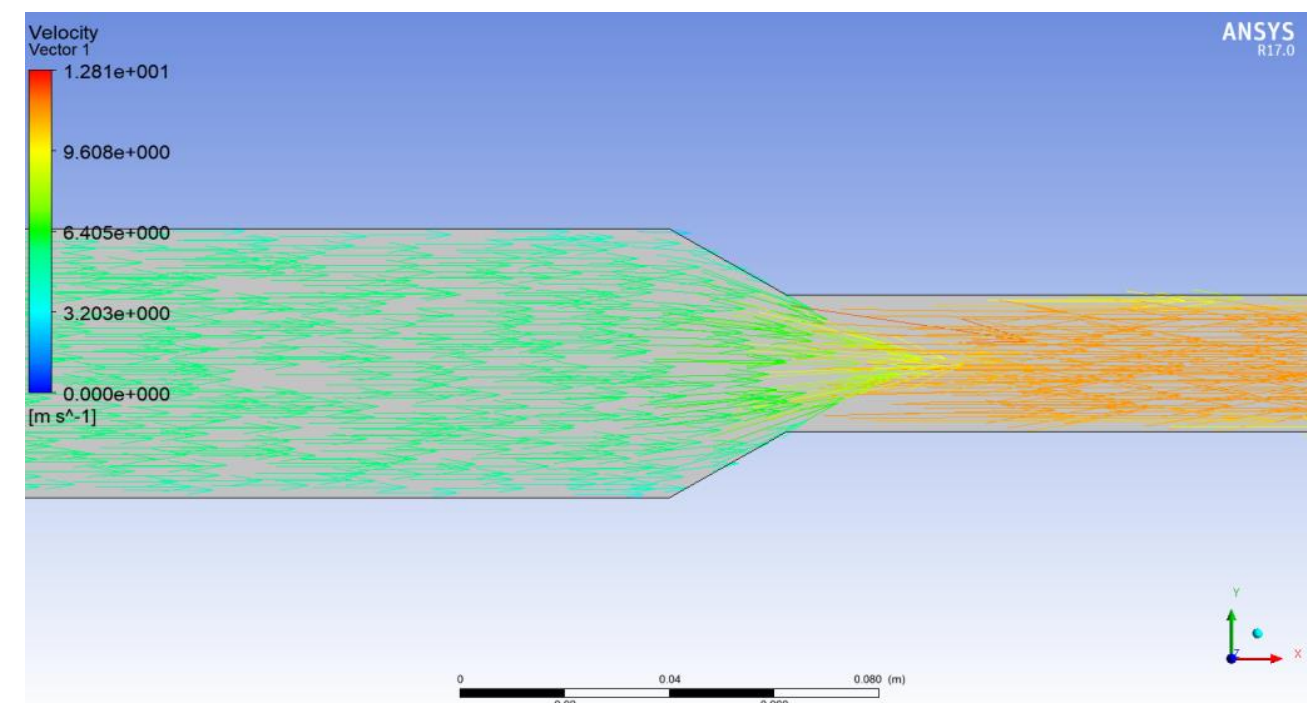

Figura 8. Vectores de velocidad

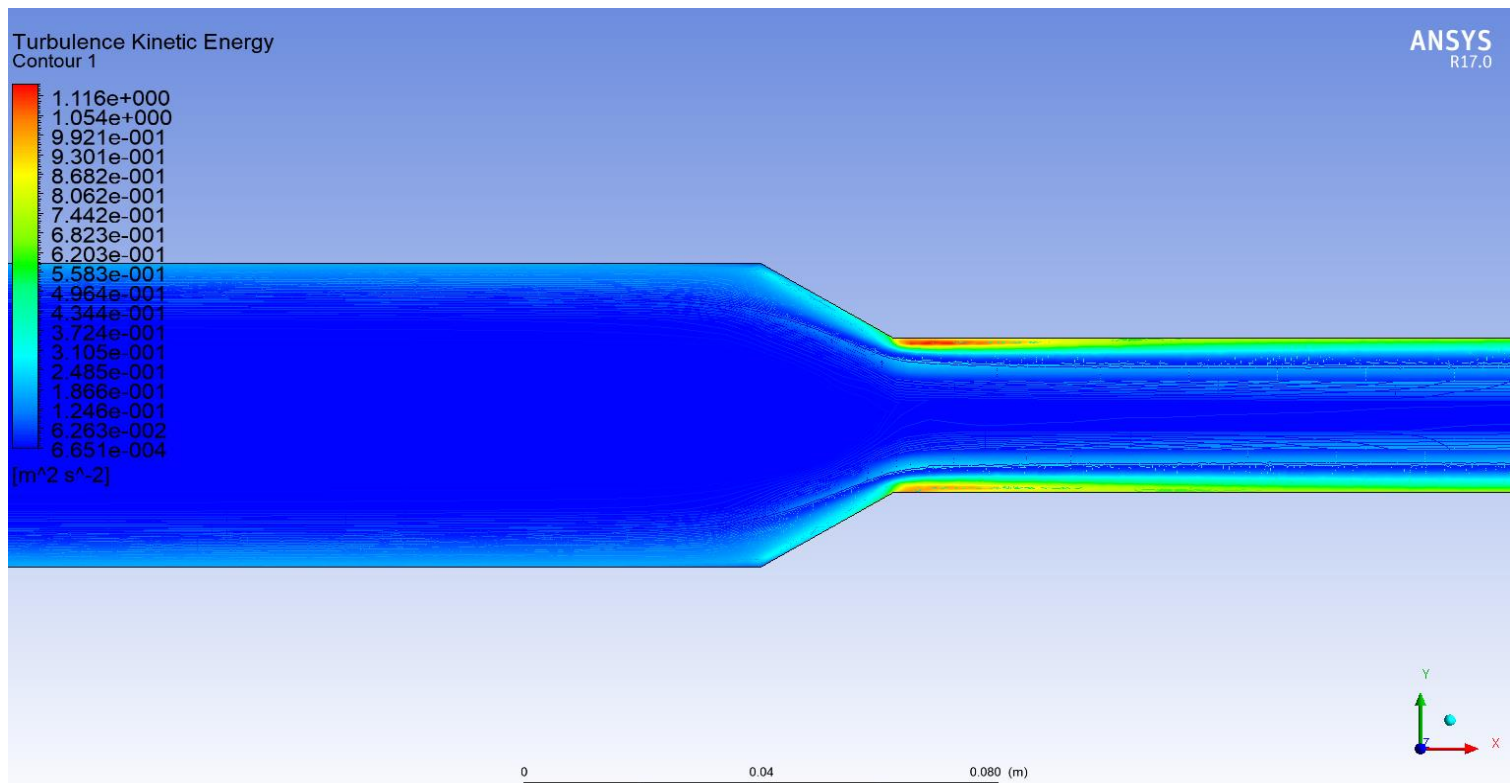

Figura 9. Turbulencia K-épsilon estándar

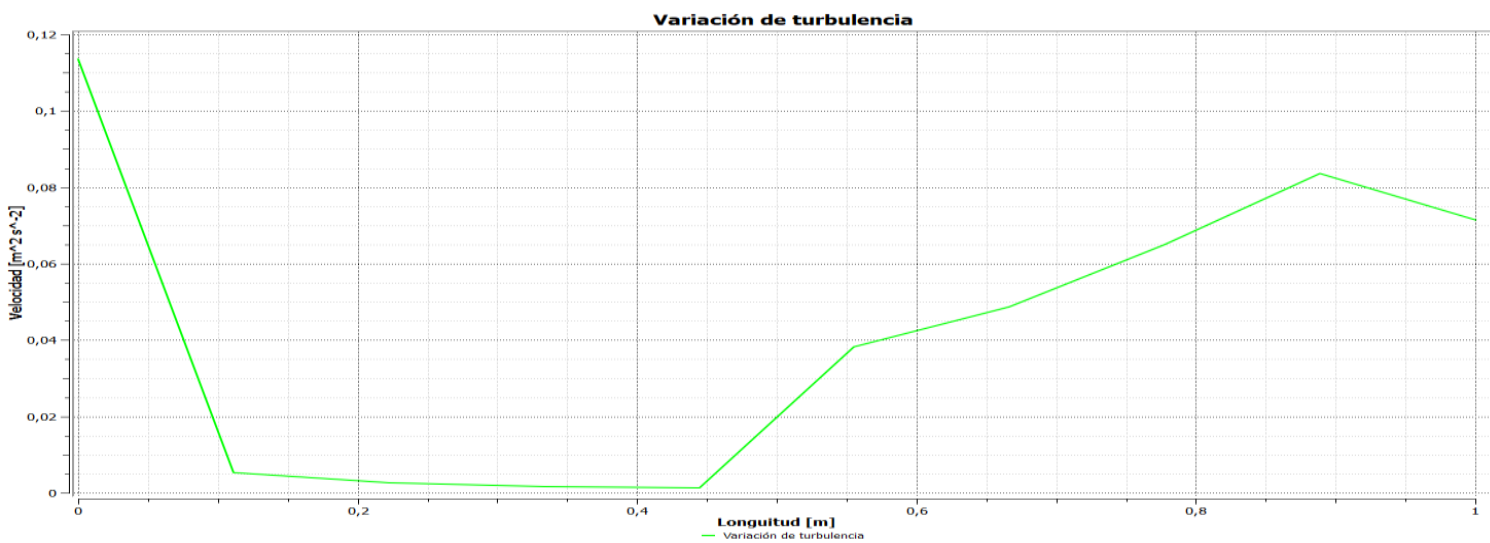

Figura 10. Variación de la turbulencia

Enfoque UTE, V.9-N.3, Sep.2018, pp. 80 - 92 
La presión, al inicio de la tubería, como se muestra en la Figura 11, se ve que es mayor y constante hasta la contracción gradual; si sabemos que hemos tomado en cuenta la presión atmosférica, esta baja debido a disminución del área transversal, manteniéndose igualmente constante después de la contracción gradual.

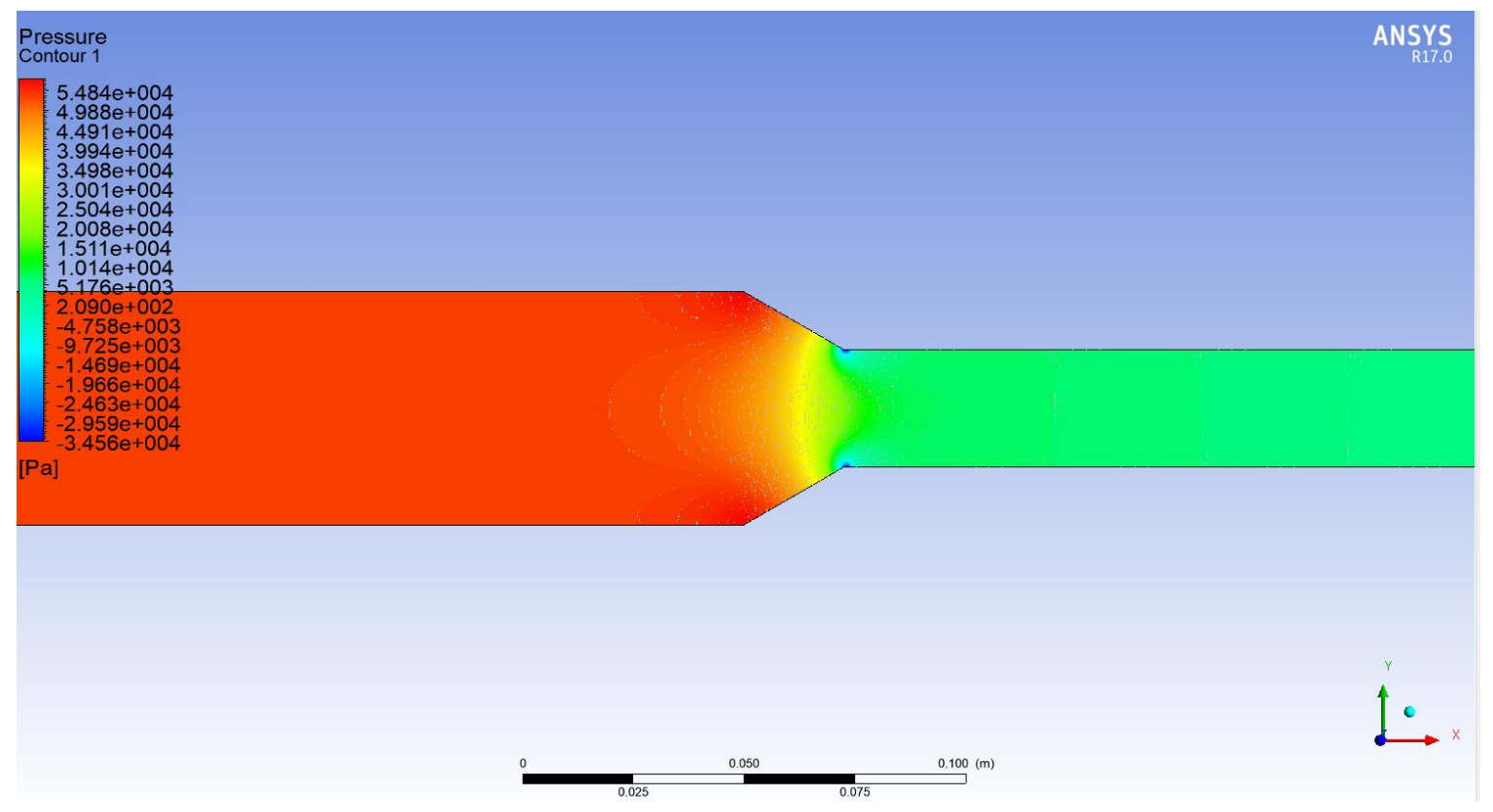

Figura 11. Presión en tubería

Se muestra en la Figura 12, la representación del cambio en la presión que se provoca antes y después de la contracción de la tubería, además se observa la fuerte caída de presión a lo largo de la tubería.

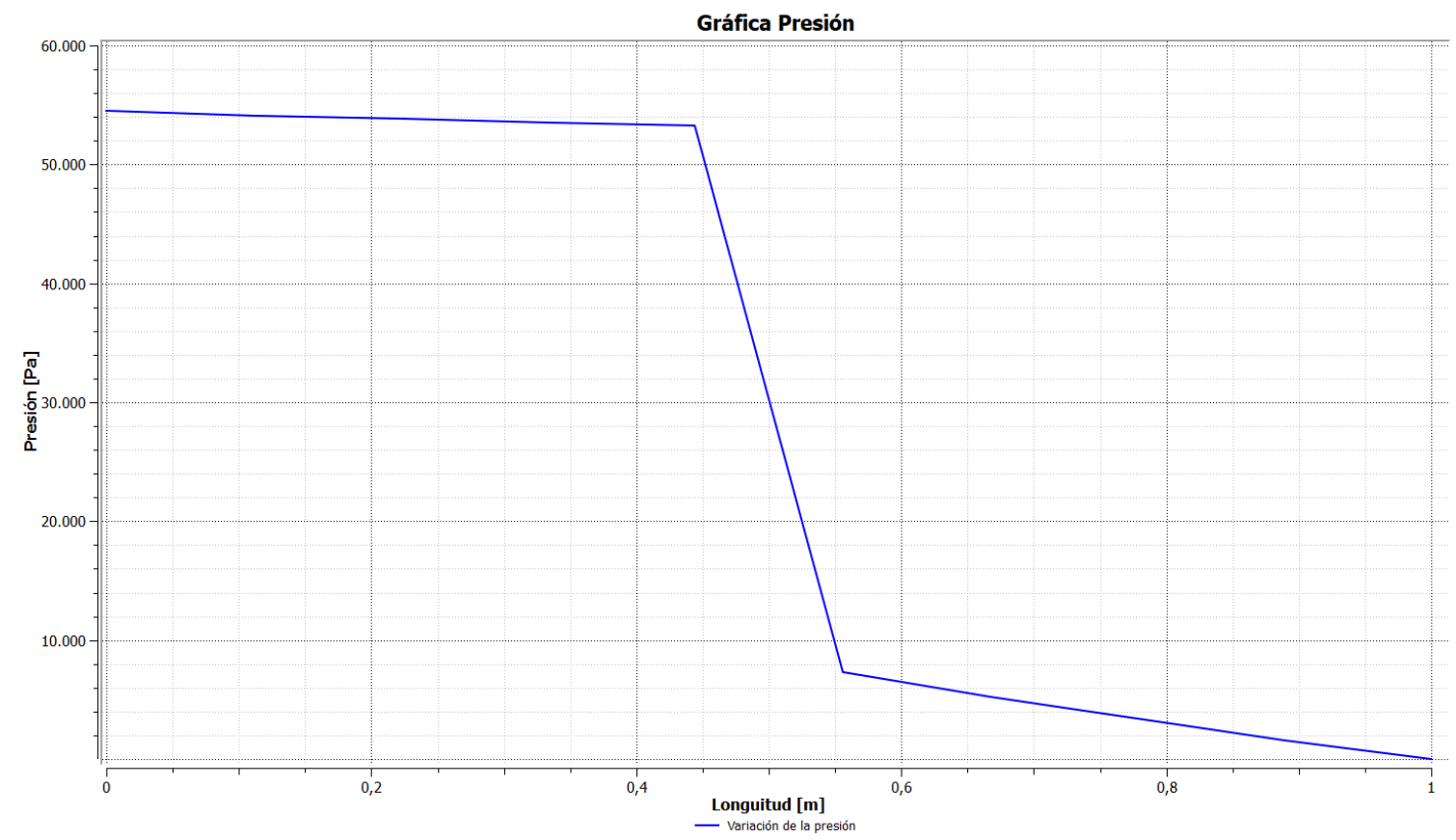

Figura 12. Variación de la presión 
Asímismo, se muestra en la Figura 13, la representación con líneas de flujo de la turbulencia que se genera antes y después de la contracción de la tubería, este fenómeno es típico cuando existen reducciones o expansiones en líneas de flujo.

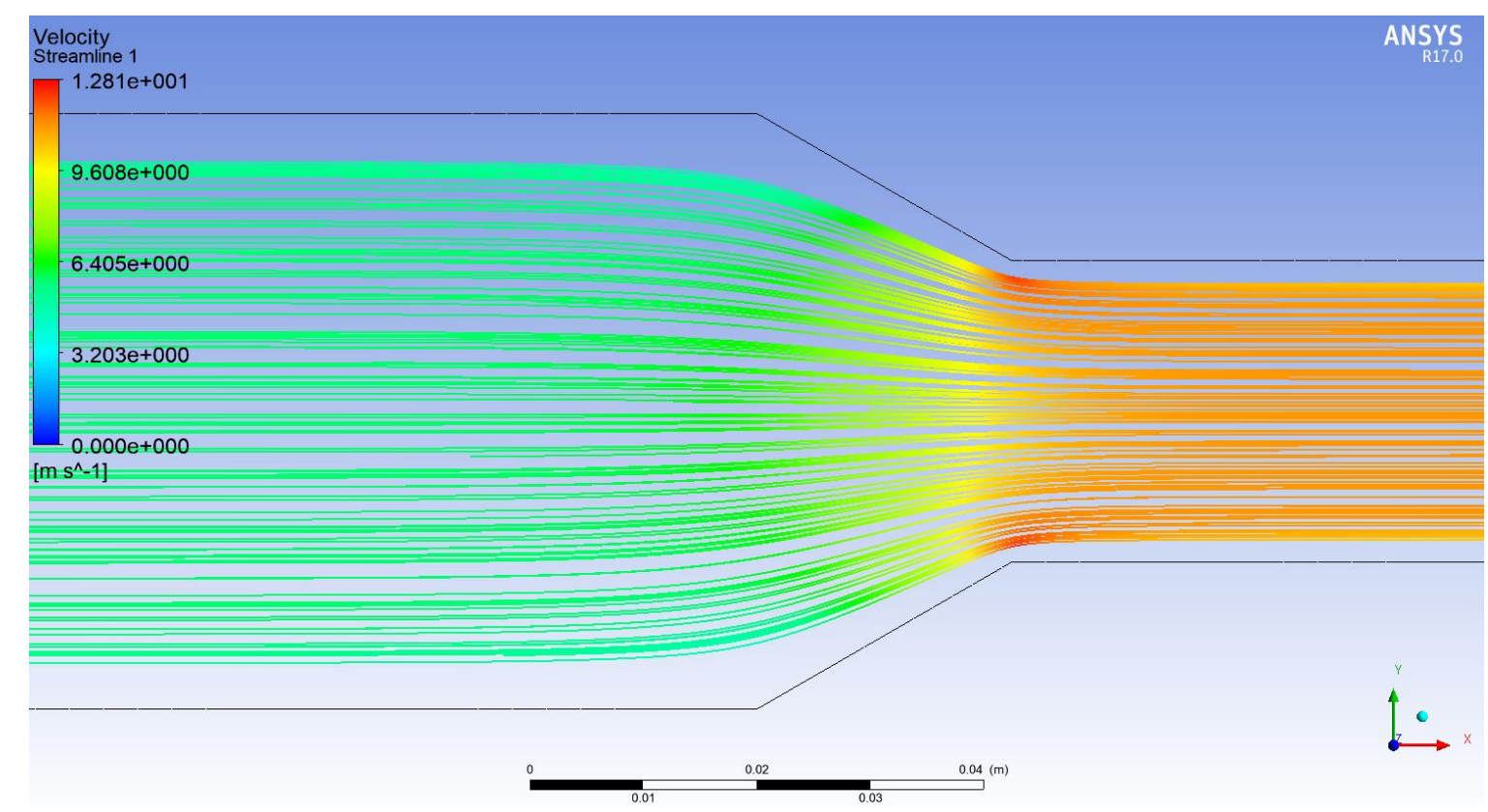

Figura 13. Línea de flujo de turbulencia

\subsection{Discusión}

El análisis realizado en este trabajo así como los resultados obtenidos y expuestos, se asemejan a los presentados por Villarroel (2015), en donde recalca que en las aguas debajo de la contracción se produce un incremento de velocidad, en las zonas aledañas a la válvula, con valores aproximados al $80 \%$ con respecto a la velocidad media, además que la recirculación de fluido se produce en las cercanías de la pared ocasionado por la turbulencia que se genera, por lo que se espera que se produzca desgaste excesivo en la zona de influencia.

Finalmente (Toro, 2012) enfatiza de la misma forma que la caída de presión se debe a la reducción del área transversal por donde circula el fluido, esto se traduce a pérdida de energía por la acción de la reducción gradual en los sistemas de tubería.

\section{Conclusiones y recomendaciones}

En función a los resultados obtenidos, se pueden plantear las siguientes conclusiones:

- Se realizó la simulación numérica para la reducción gradual, con diámetro nominal de $0.50 \mathrm{~mm}$, diseñada previamente en INVENTOR, mediante el software ANSYS CDF 17.0. La simulación se elaboró en estado estacionario, para un fluido con velocidad de entrada de $5.5 \mathrm{~m} / \mathrm{s}$ y una temperatura de $25^{\circ} \mathrm{C}$.

- En cuanto al perfil de velocidades del flujo, se determinó que la velocidad aumenta en un $140 \%$ más que la velocidad inicial debido a la contracción gradual.

- El flujo a través de una contracción gradual fue numéricamente simulado con un flujo de agua que atraviesa toda la tubería, mediante el modelo de turbulencia kappa-épsilon y se estableció que la turbulencia se acrecentó a la salida de la contracción, por lo cual este tipo de zonas se deben considerar para fines de mantenimiento. 
- Igualmente, los resultados de esta investigación indican que fluent se puede usar con un alto grado de precisión, cerca del $95 \%$, para visualizar y comprender las caídas de presión debido a los accesorios que poseen las líneas de tuberías, así como interpretar de mejor manera el cambio de las propiedades de este tipo de fluido.

- Finalmente, las pérdidas de energía son menores en el punto donde comienza la reducción en la tubería. La presión disminuye con una mayor entrada de área hacia una reducción de sección, por lo tanto, con mayor tasa de flujo másico en la contracción. Este es el punto más susceptible para daño de la tubería. Luego, para aumentar la vida de la tubería en casos de repentina contracción y ampliación, las tuberías deben diseñarse en vista de las observaciones anteriores.

\section{Bibliografía}

Anaya-Durand, A., Cauich-Segovia, I., Funabazama-Bárcenas, I., \& Gracia-MedranoBravo, O. (2014). Evaluación de ecuaciones de factor de fricción explícito. Revista Mexicana Ingeniería Química, II(25), 128-134.

ANSYS. (s.f.). ANSYS CFX Release Notes for 14.0.

Bae, Y., \& Kim, Y. (2014). Prediction of local loss coefficient for turbulent flow in axisymmetric sudden expanexpansions. Effect of Reynolds number. Journal Annals of Nuclear Energy(73), 33-38.

Bardina, J. E., Huang, P. G., \& Coakley, T. J. (1997). Turbulence Modeling Validation Testing and Development. NASA Technical Memorandum (110446).

Bariviera, D., Frizzone, J., \& Rettore, A. (2013). Dimensional analysis approach to estimate local head losses in microirrigation connectors. Journal Irrigation Science, 3(32), 169179.

Binding, D., Phillips, P., \& Phillips, T. (2006). Contraction/expansion flows: The pressure drop and related issues . Journal of Non-Newtonian Fluid Mechanics(137), 31-38.

Elbatran, A., Yaakob, H., \& Ahmeda, B. (2015). Operation, performance and economic analysis of low head micro-hydropower turbines for rural and remote areas. Renewable and Sustainable Energy(43), 43-50.

Garzón Cruz, J. C. (2014). Universidad ElA. Obtenido de http://fluidos.eia.edu.co/hidraulica/articuloses/flujoentuberias/reducci\%C3\%B3n/redu cci\%C3\%B3n.htm

F. M. (2000). Design and off design pipe network geothermal power plant analysis with power pipe simulator. Energy Conversion \& Management, XLI(12), 1223-1235.

Fester, V., Mbiya, B., \& Slatter, P. (2008). Energy losses of non-Newtonian fluids in sudden pipe contractions. Chemical Engineering Journal(145), 57-63.

Fox, R., Pritchard, P., \& Mcdonald, J. (2006). Introduction To Fluid Mechanics. Usa: ohn Wiley \& Son Inc.

Franzini, J., \& Finnemore, E. (1999). Mecánica de fluidos,con aplicaciones en ingeniería. España: Mc Graw-Hill, Inc.

Mataix, C. (2010). Mecánica de Fluidos y Máquinas Hidráulicas. México: Editorial Alfaomega.

Menter, F. (1994). Two-equation eddy-viscosity turbulence models for engineering. AIAAJournal, 32(8), 1598-1605.

Montilva, M. (2009). Flujo Laminar en la Región de entrada de un tubo recto precedido por una tuberia curva. Venezuela.

Mott, R. L. (2006). Pérdidas Menores. En Mecánica de Fluidos (págs. 279-320). México: Pearson Educación.

Pedros, R. (2010). Densidad y Viscosidad. España: AISC.

Rend, R., Sparrow, E., Bettenhausen, D., \& Abraham, J. (2013). Parasitic pressure losses in diffusers and in their downstream piping systems for fluid flow and heat transfer. International Journal of Heat and Mass Transfer(61), 56-61. 
Schiller, L. (1992). Die Entwichklung der Laminaren Geschwindigkeitsvertlung und ihre Bedeutung fur. Rusia: Z Angew Math,.

Sesma, J., Molina-Martínez, J., Cavas-Martínez, F., \& Fernández-Pacheco, D. (2015). A mobile application to calculate optimum drip irrigation laterals. Agricultural Water Management(151), 13-18.

Sotelo, A. G. (2013). Hidráulica General. Hidráulica General: LIMUSA.

Streeter, V., Wylie, E., \& Bedford, K. (2000). Mecánica de fluidos. Bogotá, Colombia: Mc Graw-Hill Internacional SA.

Toro, A. D. (2012). COMPUTATIONAL FLUID DYNAMICS ANALYSIS OF BUTTERFLY VALVE PERFORMANCE FACTORS. Logan, Utah.

Unet, U. d. (2016). Flujos en tuberias, Flujos Internos. Caracas: AISC. Obtenido de http://www.unet.edu.ve/ fenomeno/F_DE_T-153.htm

Villaroel Quinde, L. (2015). Pontificia Universidad Católica del Perú. Recuperado el 2015, de http://tesis.pucp.edu.pe/repositorio/handle/123456789/6408

Villarroel Quinde, L. F. (2015). Simulación numérica de un flujo de agua a través de una válvula tipo mariposa de doble excentricidad. Lima - Perú.

Yildirim, G., \& Shing, V. P. (2010). A MathCAD procedure for commercial pipeline hydraulic design considering local energy losses. Advances in Engineering Software(41), 489496. 